\title{
Sparsity Independent Sub-Nyquist Rate Wideband Spectrum Sensing on Real-time TV White Space
}

\author{
Yuan Ma, Student Member, IEEE, Yue Gao, Senior Member, IEEE, Andrea Cavallaro, Member, IEEE, \\ Clive G. Parini, Member, IEEE, Wei Zhang, Fellow, IEEE, and Ying-Chang Liang, Fellow, IEEE
}

\begin{abstract}
Wideband spectrum sensing is a highly desirable feature in cognitive radio systems when the aim is to increase the probability of exploring spectral opportunities. Sub-Nyquist sampling has attracted significant interests for wideband spectrum sensing, while existing algorithms can only work with a sparse spectrum. In this paper, we propose a sub-Nyquist wideband spectrum sensing algorithm that achieves wideband sensing independent of signal sparsity without sampling at full bandwidth by using the low-speed analog-to-digital converters based on sparse Fast Fourier Transform. To lower signal spectrum sparsity while maintaining the channel state information, we pre-process the received signal through a proposed permutation and filtering algorithm. The proposed wideband spectrum sensing algorithm sub-samples the time-domain signal and then directly estimates its frequency spectrum. We derive and verify the proposed algorithm by numerical analysis and test it on real-world TV white space signals. The results show that the proposed algorithm achieves high detection performance on sparse and non-sparse wideband signals with reduced runtime and implementation complexity in comparison with the conventional wideband spectrum sensing algorithms.
\end{abstract}

Index Terms-Wideband Spectrum Sensing, Sub-Nyquist Sampling, sparse Fast Fourier Transform, TV White Space.

\section{INTRODUCTION}

$\mathbf{C}$ OGNITIVE radio algorithms opportunistically access the underutilized spectrum by seeking and utilizing temporarily vacant frequency bands [2], [3]. To enable dynamic spectrum access, sensing the spectrum before transmission is an important stage for the secondary user (SU) in order to detect the presence of the primary user (PU). Accurate spectrum sensing allows unlicensed SUs to access the unused spectrum opportunistically, without causing interference to the transmissions of the licensed PUs.

To increase the probability of finding unutilized spectrum, wideband spectrum sensing is highly desirable for cognitive radio systems [4]. An important application of wideband spectrum sensing is the cognitive access to the unused portions of the UHF spectrum in the TV bands, the TV white space

Yuan Ma, Yue Gao, Andrea Cavallaro and Clive G. Parini are with the School of Electronic Engineering and Computer Science, Queen Mary University of London, London E1 4NS, U.K. (emails:\{y.ma, yue.gao, a.cavallaro, c.g.parini\}@qmul.ac.uk).

Wei Zhang is with the School of Electrical Engineering and Telecommunications, University of New South Wales, Sydney, N.S.W., Australia (e-mail: wzhang@ee.unsw.edu.au).

Ying-Chang Liang is with the University of Electronic Science and Technology of China, Chengdu 611731, China, and also with the University of Sydney, Australia (e-mail: liangyc@ieee.org).

Part of this paper is presented at in the IEEE International conference on World of Wireless, Mobile and Multimedia Networks (WoWMoM), Boston, MA, Jun. 2015 [1].
(TVWS), which is composed of these channels that are not used by digital terrestrial television (DTT) or programme making and special events (PMSE) users, and those freed up by the switch-over from analogue to digital TV broadcasting [5], [6]. Compact and low power white space devices for rural broadband/WiFi-like accesses and Machine-to-Machine (M2M) communications could therefore be operated by SUs on these vacant channels without interfering with the primary transmissions [7], [8].

Fast and accurate detection of the surrounding spectrum is crucial to enable dynamic spectrum access over TVWS that does not cause interferences to PUs [9], [10]. One major current approach to discover available TVWS channels is using a Geo-location database [5]. However, this approach requires an initial wired or wireless link available at the master white space device in order to report its location to the central database. Moreover, rapid dynamic changes of the wireless environment pose significant challenges to this database-only approach. Dynamic spectrum sensing and its combination with database approaches could address these challenges [11].

In the wideband regime, a major challenge arises from the stringent requirements on the high sampling rate at the analogto-digital converters (ADCs) to transform the received signal into a digital signal by sampling at the Nyquist rate [4]. In [12], Quan et al. proposed a multiband joint detection scheme that can sense the primary transmissions over multiple frequency bands simultaneously. Furthermore, Tian and Giannakis proposed a wavelet-based wideband spectrum sensing algorithm, which exploits wavelet transform to scan through the wide bandwidth to identify all piecewise smooth subbands [13]. However, these algorithms require high sampling rate in order to characterize the entire wide bandwidth, which presents significant challenges in the high-speed sampling hardware and signal processing algorithms.

A simple approach to relax the high sampling rate requirement for wideband spectrum sensing is to use a tunable narrowband bandpass filter at the radio-frequency (RF) front-end to scan through all of the narrow channels one by one to detect the existence or non-existence of licensed primary transmissions [14], [15]. However, the sequential nature of such schemes could introduce a long sensing period. Such delay in the sensing process will also cause missed opportunities or interferences to PUs. In [16], a filter bank algorithm was proposed to process the wideband signal by multiple narrowband bandpass filters with different shifted central frequencies. However, it requires a great number of RF front-end components, e.g., bandpass filters, ADCs, and 
therefore significantly increases the cost.

Sub-Nyquist sampling techniques implement wideband spectrum sensing using lower-than-Nyquist sampling rates to reduce the requirements of high-speed signal processing [17]. Compressed sensing (CS) can be used to implement wideband sensing by exploiting the sparseness of the wideband signal in the frequency domain [18]. CS-based wideband sensing has attracted considerable attention for wideband signal acquisition because it uses fewer measurements [19]-[25]. However, CS based approaches require random sub-Nyquist projections of the wideband signals [26], which cannot be done simply by using the standard low-rate ADCs. Therefore, custom ADCs with complex hardware that can perform analog mixing or analog matrix multiplication at Nyquist rate are needed in compressive wideband spectrum sensing schemes [27]-[29].

In this paper, we present a novel sub-Nyquist wideband spectrum sensing algorithm based on sparse Fast Fourier Transform (sFFT) [30]. To lower signal spectrum sparsity while maintaining the channel state information, we propose a permutation and filtering algorithm to pre-process the received signal. The proposed algorithm works for both sparse and nonsparse spectrum without sampling at full bandwidth through the use of low-speed ADCs. Based on the proposed permutation and filtering algorithm, sub-Nyquist wideband sensing can be implemented even when the signal is highly occupied by the PUs. Unlike existing sub-Nyquist approaches [18], [19], the proposed algorithm sub-samples the wideband signal independent of signal sparsity and detects the channel occupancy state based on the estimated spectrum. We derive and verify by numerical analyses the mathematical model of the proposed spectrum sensing algorithm. Moreover, we test the proposed algorithm on real-world TVWS signals. This validation shows reduced runtime and implementation complexity as well as high detection performance compared with the conventional wideband sensing algorithms.

The rest of this paper is organized as follows: Section II discusses the prior work on spectrum sensing. In Section III, we present the proposed wideband spectrum sensing algorithm and each block in the model. The simulations for the performance evaluation of the proposed sensing scheme are presented in Section IV on the simulated and real-world TVWS signals. Finally, conclusions are drawn in Section V.

\section{PRIOR WORK}

According to the bandwidth of the spectrum of interests, spectrum sensing can be categorized into two types, narrowband spectrum sensing and wideband spectrum sensing [4]. Here, the term narrowband implies that the frequency range is sufficiently narrow such that the channel frequency response can be considered as flat.

Traditional narrowband spectrum sensing techniques can be categorized in three groups, namely matched filtering detection [31], energy detection [32] and cyclostationary feature detection [33]. The advantages and disadvantages of these algorithms are summarized in Table I.

While narrowband spectrum sensing aims to explore spectral opportunities over narrow frequency range, cognitive radio networks will eventually be required to exploit spectral opportunities over a wide frequency range to find more spectral opportunities and achieve higher opportunistic throughput. As shown in Table I, wideband spectrum sensing can be broadly divided into two types: Nyquist wideband sensing and subNyquist wideband sensing. Nyquist wideband sensing processes received signals taken at or above the Nyquist rate, which leads to unaffordable high sampling rate and implementation complexity. Sub-Nyquist sampling technique therefore attracts more and more attention to achieve a more flexible and faster wideband spectrum sensing, such as compressive sensing and sFFT, etc.

Compressed-sensing based spectrum sensing acquires wideband signals using the sampling rates lower than the Nyquist rate and detects the spectral opportunities using these compressed measurements. Most CS-based sensing algorithms typically assume that the wideband signal is sparse in the frequency domain given the low spectrum utilization, e.g., $3 \%$ in [22], $8 \%$ in [19], $10 \%$ in [23], 25\% in [24], and $30 \%$ in [18]. As the secondary market for the spectrum sharing has been opened to public usage, multiple SUs compete for the TVWS spectrum resources to serve a large pool of end-users [5]. Since the usage patterns may change from day-to-day or even from hour-to-hour, there is a wide range of variations in the spectrum occupancy due to the secondary signals vacating or coming into existence [34], [35]. Therefore, the wideband signal may no longer be static and sparse over TVWS, i.e., sparsity level may be time-varying and larger than $30 \%$ [36], [37].

The sFFT algorithm estimates the spectrum of a sparse signal directly from the time-domain sub-Nyquist samples [30]. Compared with DFT, sFFT reports only the positions and amplitudes of the large magnitude frequency components of the input signal in the time proportional to the sparsity of the signal spectrum, as opposed to the signal size. As spectrum sensing focuses on clarifying the positions of the occupied frequency bands, it can benefit from the advances of sFFT, which permit signals whose frequency domain representation is sparse to be recovered using only a small subset of samples [30].

However, the performance of the sFFT has strict requirements on the input signal sparsity, which limits its application to only very sparse signals. sFFT outperforms FFT only when the number of non-zero dominant frequency components $\kappa$ is less than $O(N / \log N)$, where $N$ is the number of frequency bins. For example, when signal size equals to $N=2^{22}$, the signal sparsity level $\kappa / N$ should be less than $0.048 \%$ [30]. However, the PU's spectrum utilization is about $20 \%$ to $30 \%$ [3], and will be higher when the secondary market is opening for the spectrum sharing to the mobile devices [36]. In [29], a wideband spectrum sensing scheme is proposed for the spectrum that is not sparse, where its goal is to identify the changes in the spectrum occupancy, based on the assumption that although the spectrum is densely occupied, its changes are sparse. However, it can only detect the spectrum changes, not the occupancy states over the whole spectrum, and has strict requirement on the percentage of the spectrum changes, up to $1 \%$. Hence a flexible and fast wideband spectrum sensing 
TABLE I

SUMMARY OF ADVANTAGES AND DISADVANTAGES OF NARROWBAND AND WIDEBAND SPECTRUM SENSING ALGORITHMS

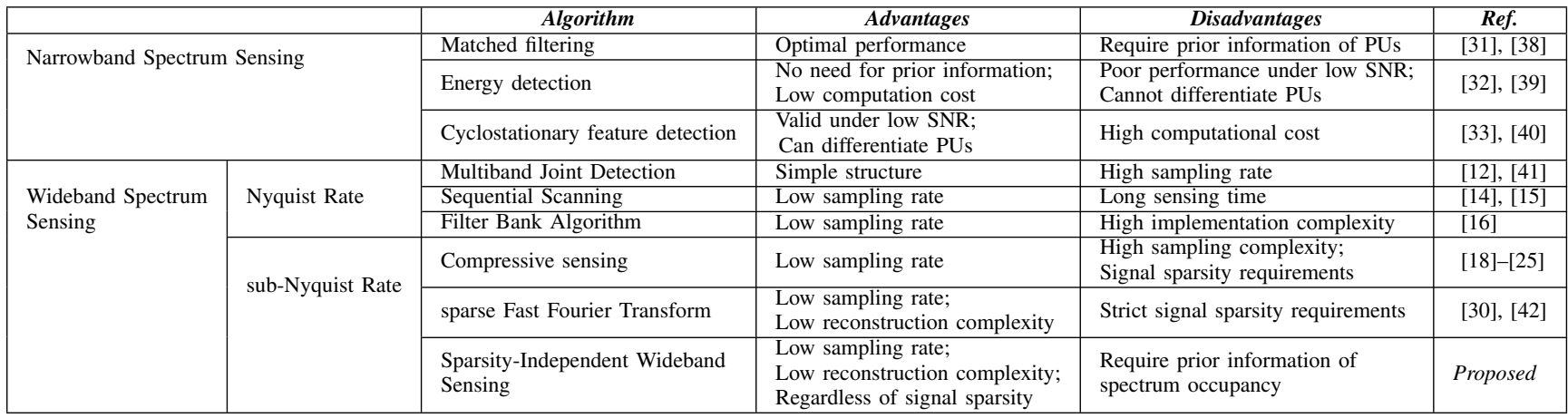

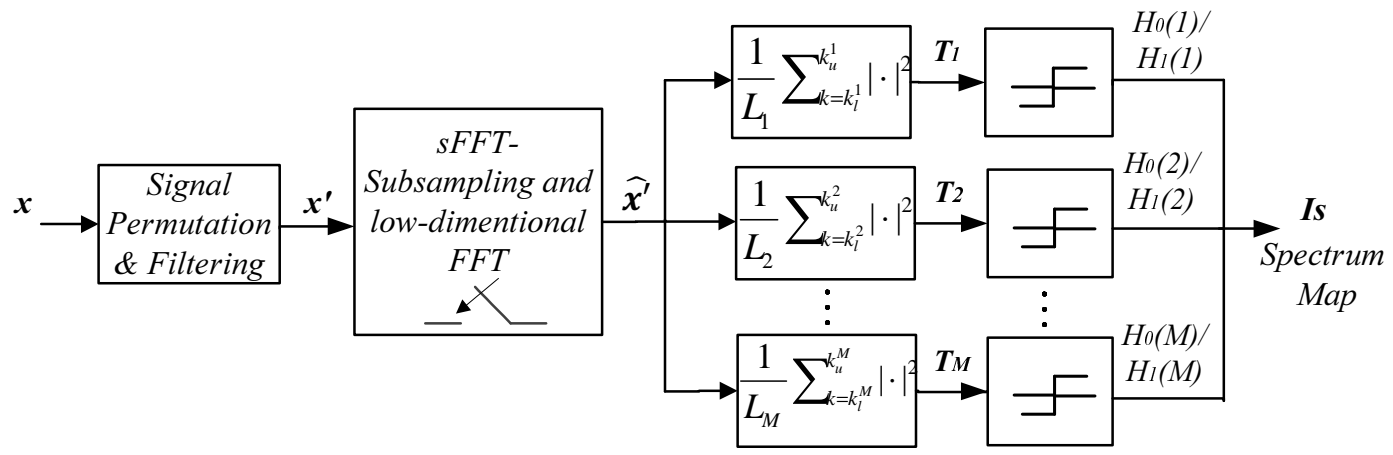

Fig. 1. Block diagram of the proposed wideband spectrum sensing scheme.

scheme that can subsample the wideband signal regardless of signal sparsity is of great interest.

\section{Permuted And Filtered Wideband Sensing BASED ON SFFT}

In this section we present the signal model and the proposed scheme for the wideband spectrum sensing, which is composed of three main blocks: signal permutation and filtering; spectrum estimation and multi-channel joint detection, as shown in Fig. 1.

\section{A. Problem formulation}

Without loss of generality, the wideband signal to be monitored is bandlimited with $M$ non-overlapping channels, each of them with bandwidth $B_{0}$. The whole bandwidth is $B=M B_{0}$. The channels are indexed from 1 to $M$. Suppose there are $S \leq M$ active channels occupied by PUs during the sensing period with $I_{S}$ denoting the set containing the indices of the occupied channels. Thus the spectrum utilization ratio of the primary transmission can be expressed as $\alpha=S / M$.

If the input signal $x$ is sampled at the Nyquist rate $f_{s}$ in the observed time $T_{s}$, the signal can be discretized as a vector $\boldsymbol{x} \in \mathbb{C}^{N}$, where $N$ denotes the number of Nyquist samples and can be written as $N=f_{s} T_{s}$. Let $s$ be the wideband primary signal expressed as $s=\sum_{m=1}^{M} s_{m}$, where $s_{m}$ is the primary signal in the channel $m$. The received signal $x$ at the cognitive radio front-end can be expressed as:

$$
x=s+\omega,
$$

where $\omega$ represents white Gaussian noise with zero mean and variance $\sigma_{\omega}^{2}$. If we denote with $k$ the frequency bin index $(k=0,1, \ldots, N-1)$, the Fourier spectrum of $\boldsymbol{x}, \widehat{\boldsymbol{x}}$, can be computed by DFT as

$$
\widehat{x}_{k}=\frac{1}{\sqrt{N}} \sum_{i=0}^{N-1} x_{i} e^{-j 2 \pi i k / N} .
$$

Since the bins of DFT are uniformly distributed across the total bandwidth, ignoring the zero frequency, each channel can be represented by the same number of frequency bins $L=$ $(N-1) / M=k_{u}^{m}-k_{l}^{m}+1$, where $k_{u}^{m}$ and $k_{l}^{m}$ are the upper and lower bin indices of channel $m$.

The goal of wideband spectrum sensing is to locate the active channel set $I_{S}$, namely to detect the presence of the primary signal in each channel of the input signal.

\section{B. Signal Permutation and Filtering}

As sFFT has strict requirements on input signal sparsity, we propose a permutation and filtering algorithm to pre-process the received signal to reduce the spectrum sparsity lower than the sparsity bound of sFFT while maintaining the channel state information. The proposed permutation and filtering algorithm is composed of three main steps, namely permutation, filtering and un-permutation (Fig. 2).

Let the extraction ratio be $\beta$ and the number of samples taken on each channel be $L_{m}=\lfloor\beta L\rfloor$. Given the spectrum utilization ratio $\alpha$, the sparsity of the input signal $\kappa=S L=$ $\alpha M L$ is reduced to $\kappa^{\prime}=S L_{m}=\alpha M L_{m} \approx \alpha \beta N$. Therefore, 


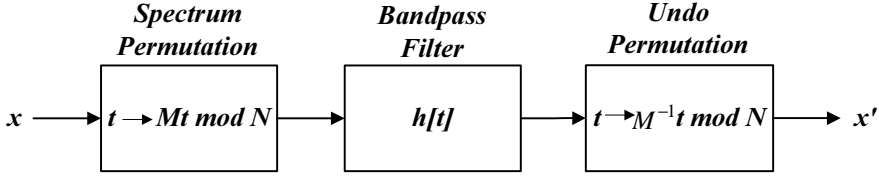

Fig. 2. Flowchart of the proposed signal permutation and filtering algorithm.

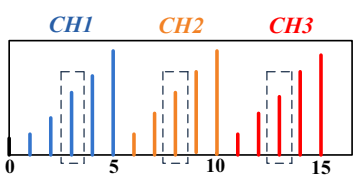

Frequency

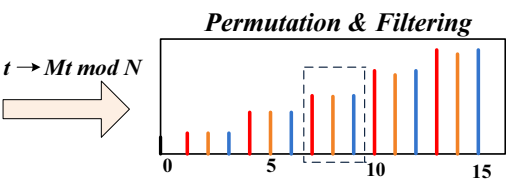

Frequency
Fig. 3. (left) Input signal spectrum with 3 channels $(N=16)$, (right) signal spectrum after permutation. Through the bandpass filter, it can extract the central frequencies in each channel.

the available range of the extraction ratio to retain faster runtime of sFFT than FFT is $\beta \leq \beta_{\max }=\frac{L_{m}}{L}=\frac{\kappa_{\max } / \alpha M}{N / M}=\frac{\kappa_{\max }}{\alpha N}$, where $\kappa_{\max }=O(N / \log N)$ is the sFFT's sparsity bound for the input signal size $N$.

Based on the input spectrum utilization $\alpha$, the extraction ratio $\beta$ can be chosen to reduce signal sparsity lower than the sFFT's sparsity bound. sFFT can then be applied to reduce the runtime and computational complexity. However, for the $M$-channel wideband signal, it needs $M$ bandpass filters to extract the subset of central samples from each channel.

To implement the extraction process with low complexity, we permute the signal through time dilation $t \longmapsto M t \bmod$ $N$ at first. As the input signal size $N=M L+1, M$ is invertible modulo $N^{1}$, where $L$ is the number of frequency components in each channel, ignoring the zero frequency, $L=$ $(N-1) / M$. In the frequency domain, this translates to the frequency mapping $f \longmapsto M^{-1} f \bmod N$, where $M M^{-1} \bmod$ $N=1$. The inverse of $M$ under $\bmod -N$ equals to $M^{-1}=$ $N-L$.

As shown in Fig. 3, the $l$-th frequencies from each of the $M$ channels $(M-m) L+l, m=1, \ldots, M$, are moved to $(l-1) M+m, l=1, \ldots, L$ in the permuted signal ${ }^{2}$. Therefore, the signal components from different channels are uniformly interleaved with each other in the frequency domain after the permutation.

Thus, we can pass the permuted signal through one bandpass filter to extract $L_{m}$ central frequencies from each channel. The bandwidth of the filter is $B_{\text {filter }}=\beta B$. Finally, the frequencies in the filter output are restored to their original spectral locations by carrying out a reverse time dilation $t \longmapsto M^{-1} t \bmod N$.

\footnotetext{
${ }^{1}$ Suppose there exists an integer $M^{-1}, M M^{-1} \bmod N=1$. There must exist an integer $a \in \mathbb{Z}$, such that $M M^{-1}=a N+1=a(M L+1)+$ $1^{a=\underline{M-1}^{-1}}(M-1) M L+M$. So $M^{-1}=(M-1) L+1=M L+$ $1-L=N-L \in \mathbb{Z}$. Hence $M$ is invertible modulo $N$ and its inverse is $M^{-1}=N-L$.

${ }^{2}[(l-1) M+m] \cdot M^{-1} \bmod N$

$=[(l-1) M+m] \cdot[N-L] \bmod N$

$=N \cdot[(l-1) M+m]-M L l+[M-m] L \bmod N$

$=N \cdot[(l-1) M+m]-l[M L+1]+[M-m] L+l \bmod N$

$=N \cdot[(l-1) M+m-l]+[M-m] L+l \bmod N$

$=[M-m] L+l$.
}

After the signal permutation and filtering, the sparsity of input signal has been lowered so that its frequency representation $\widehat{\boldsymbol{x}}^{\prime}$ can then be estimated through sFFT.

\section{Spectrum Estimation}

Based on the permuted and filtered signal $\boldsymbol{x}^{\prime}$, we then subsample it and estimate its spectrum $\widehat{\boldsymbol{x}}^{\prime}$ based on sFFT [30].

The key idea behind sFFT is to identify the positions and amplitudes of the large magnitude frequency components based on the partial measurements from its subsampling process. For $\boldsymbol{x}^{\prime} \in \mathbb{C}^{N}$ with a $\kappa^{\prime}$-sparse spectrum, it runs two kinds of loops to estimate the spectrum $\widehat{\boldsymbol{x}}^{\prime}$ : location loops (to find positions of $\kappa^{\prime}$ largest frequencies) and estimation loops (to estimate their magnitudes).

The purpose of the location loops is to generate a list of candidate coordinates $I_{J} \subset[N]$ which are likely to contain large magnitude frequency coefficients. Given the set $I_{J}$, estimation loops are used to determine the magnitude of the frequency coefficients. The steps below are performed in location and estimation loops :

1) Random Permutation: Randomly choose an integer $\sigma$ that is invertible modulo $N$, i.e. $\operatorname{gcd}(\sigma, N)=1$, and $\tau \in[N]$. Permute the vector $\boldsymbol{x}^{\prime}$ with the permutation $P_{\sigma, \tau}$ as

$$
\left(P_{\sigma, \tau} x^{\prime}\right)_{i}=x_{\sigma i+\tau}^{\prime}
$$

The purpose of this permutation is to rearrange signal's spectrum by modifying its time-domain permutation.

2) Filtering: Using a flat window function $F$, obtained from convolving a standard window function $G$ with a "box car" window function $H$, i.e., $\widehat{F}=\widehat{G} * H$, compute the filtered vector as

$$
\boldsymbol{y}=F \cdot\left(P_{\sigma, \tau} \boldsymbol{x}^{\prime}\right)
$$

It allows a subset of signal with size $\omega=|\operatorname{supp}(F)|$ to be extracted for computation without touching all the elements to achieve sub-linear runtime. In the frequency domain, the multiplication is equivalent to a convolution of $\widehat{F}$ and $\widehat{P_{\sigma, \tau} \boldsymbol{x}^{\prime}}$. Random time-domain permutation in the first step changes the set of coefficients binned into the filter in this step.

3) Subsampling and low-dimensional FFT: Based on a parameter $B$ dividing $N$, a low-dimensional spectrum vector $\widehat{\boldsymbol{z}}$ is computed to estimate the original spectrum, where $\widehat{\boldsymbol{z}}$ is the rate $B$ subsampling of $\widehat{\boldsymbol{y}}$, i.e., $\widehat{z}_{j}=\widehat{y}_{j \cdot(N / B)}$.

Based on the basic property of the Fourier transform: subsampling in the frequency domain causes aliasing in the time domain [43], $\widehat{z}$ is the DFT of $z_{i}=\Sigma_{j=0}^{\omega / B-1} y_{i+B j}$. Therefore, $\widehat{\boldsymbol{z}}$ can be obtained through subsampling the filtered signal $\boldsymbol{y}$ via $B$ low-speed ADCs with a decimated sampling rate $f_{s} / B$, summing it up, and then computing the $B$-dimensional DFT.

Define the "hash function" $h_{\sigma}:[N] \rightarrow[B]$ that maps each of the orignal signal coordinates to one of the $B$ "bins" in $\widehat{\boldsymbol{z}}$ as $h_{\sigma}(j)=\operatorname{round}(\sigma j B / N)$, and the corresponding offset $O_{\sigma}:[N] \rightarrow[-N /(2 B), N /(2 B)]$ to be $O_{\sigma}(j)=$ $\sigma j-h_{\sigma}(j)(N / B)$ 
4) Location Loops: Only select the top $d \cdot \kappa^{\prime}$ coordinates $h_{\sigma}(j) \in[B]$ of maximum magnitude in $\widehat{z}$ that are likely to contain large coefficients. All elements of $\widehat{y}$ that are closest to those chosen coordinates in $\widehat{z}$ will be selected for estimation and kept as the set $J$, which has the size of $d k N / B$. Reverse the coordinates in $J$ to their original positions in $\widehat{\boldsymbol{x}}^{\prime}$ and output this set as $I_{J}$, i.e.,

$$
I_{J}=\left\{j \in[N] \mid h_{\sigma}(j) \in J\right\} .
$$

5) Estimation Loops: After running $\mathcal{L}=O(\log N)$ iterations of the location loops, count the number of occurrences of each found coordinate $j$ and only keep the coordinates which occurred in at least half of the location loops as set $I_{J}^{\prime}$. For $j \in I_{J}^{\prime}$, run $\mathcal{L}$ estimation loops to estimate each frequency coefficient $\widehat{x}_{j}^{\prime}$ as

$$
\widehat{x}_{j}^{\prime}=\widehat{z}_{h_{\sigma}(j)} \omega^{\tau j} / \widehat{F}_{O_{\sigma}(j)} .
$$

Finally, it estimates all frequencies by taking the median value of $\widehat{x}_{j}^{\prime}$ on multiple estimation loops.

To balance the cost of the coordinate selection and estimation step, the dimension of $\widehat{z}$ should be selected somewhat larger than $O\left(\kappa^{\prime}\right)$ [30]. Specifically, $B$ is chosen as $B \approx \sqrt{N \kappa^{\prime}}$ to achieve the sublinear runtime $O\left(\log N \sqrt{N \kappa^{\prime} \log N}\right)$, faster than FFT to compute the spectrum for current signal sparsity $\kappa^{\prime}$ up to $O(N / \log N)$.

\section{Multi-channel Joint Detection (MJD)}

The wideband spectrum $\widehat{\boldsymbol{x}}^{\prime}$ is then divided into a series of narrowband spectra $\left\{\widehat{\boldsymbol{x}}^{\prime}[1], \ldots, \widehat{\boldsymbol{x}}^{\prime}[M]\right\}$. We jointly detect the received signal level over multiple frequency channels, which is to determine the presence or absence of the PU in each channel.

To perform binary hypothesis testing on each channel, we decouple the signal in each channel based on the estimated spectrum $\widehat{\boldsymbol{x}}^{\prime}$. According to Parseval's Theorem [44], the signal power can be calculated by adding the squared FFT bins. Thus, we compute the test statistics $T_{m}$ of channel $m$ as

$$
T_{m}=\frac{1}{L_{m}} \sum_{k=k_{l}^{m}}^{k_{u}^{m}}\left|\widehat{x}_{k}^{\prime}\right|^{2} \underset{H_{0}}{\stackrel{H_{1}}{\gtrless}} \lambda, \quad m=1, \ldots, M,
$$

where $L_{m}$ is the number of extracted samples taken on each channel after permutation and filtering, $H_{0}$ and $H_{1}$ denote the hypothesis PU absent and PU present respectively and $\lambda$ is the threshold above which $H_{1}$ is declared.

Based on Central Limit Theorem, the test statistic $T_{m}$ of channel $m$ approximately follows the Gaussian Distribution [45]:

$$
\begin{aligned}
T_{m} & \sim \mathcal{N}\left(\sigma_{\omega}^{2}, 2 \sigma_{\omega}^{4} / L_{m}\right), & & H_{0}(m) \\
T_{m} & \sim \mathcal{N}\left((1+\gamma) \sigma_{\omega}^{2}, 2(1+\gamma)^{2} \sigma_{\omega}^{4} / L_{m}\right), & & H_{1}(m),
\end{aligned}
$$

where the channel index $m=1, \ldots, M$ and $\gamma$ is the received SNR at the SU.

The performance of the detection scheme can be evaluated by two metrics: probability of detection $P_{d}$ and probability of false alarm $P_{f} . P_{d}$ is the probability of correctly detecting the

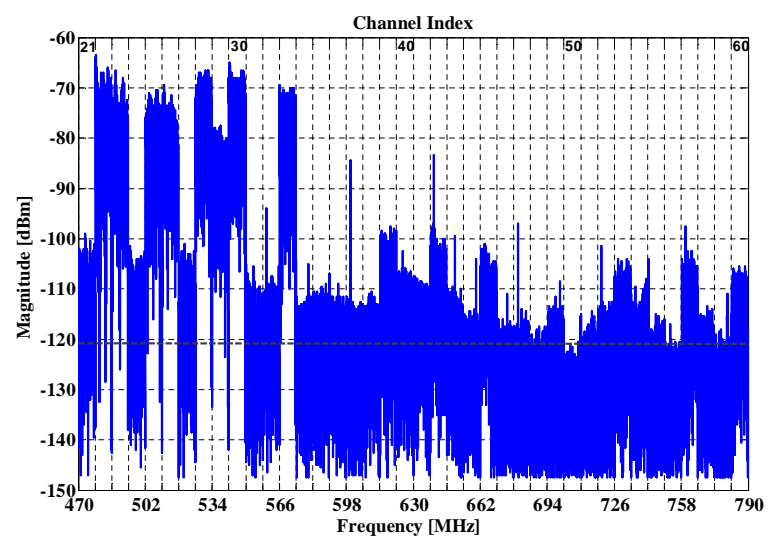

(a)

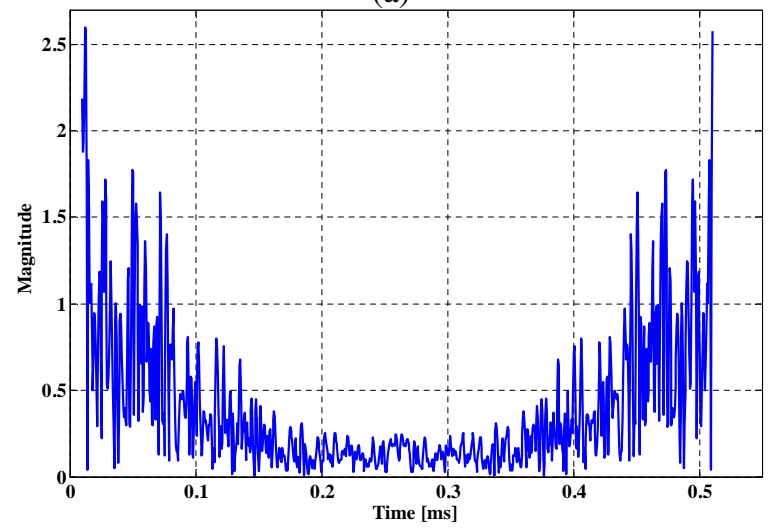

(b)

Fig. 4. (a) The power spectrum density (PSD) and (b) real-time TVWS signal recorded at $\left(51.523021^{\circ} \mathrm{N} 0.041592^{\circ} \mathrm{W}\right)$.

existence of PU on the sensing sub-channel when it is truly present and thus can be formulated as

$$
P_{d}=P\left(T_{m}>\lambda \mid H_{1}\right)=Q\left(\frac{\lambda-\sigma_{\omega}^{2}(\gamma+1)}{\sigma_{\omega}^{2}(\gamma+1) \sqrt{\frac{2}{L_{m}}}}\right) .
$$

$P_{f}$ is the probability of falsely testing that the considered channel is occupied by PU when it is actually not, and can be computed as

$$
P_{f}=P\left(T_{m}>\lambda \mid H_{0}\right)=Q\left(\frac{\lambda-\sigma_{\omega}^{2}}{\sigma_{\omega}^{2} \sqrt{\frac{2}{L_{m}}}}\right),
$$

where $Q(x)$ is the standard Gaussian Complementary Distribution Function (CDF).

To achieve the predefined false alarm probability $P_{f}$, the threshold $\lambda$ in each channel is set as

$$
\lambda=\left[\sqrt{\frac{2}{L_{m}}} Q^{-1}\left(P_{f}\right)+1\right] \cdot \sigma_{\omega}^{2},
$$

where $Q^{-1}(\cdot)$ denotes the inverse complementary distribution function of the standard normal distribution.

\section{E. Application to real-world TVWS signals}

The proposed wideband spectrum sensing scheme is further extended to real-world TVWS signal detection. The TVWS 
ranges from 470 to $698 \mathrm{MHz}$ in North America and the bandwidth of each channel is $6 \mathrm{MHz}$, while it ranges from 470 to $790 \mathrm{MHz}$ and the channel bandwidth is $8 \mathrm{MHz}$ in Europe. The setting is consistent with the current bandwidth used in TV broadcasting. For a signal with a certain total bandwidth and extraction ratio, its signal size $N$ and sparsity level after the proposed permutation and filtering scheme $\kappa^{\prime}=\alpha \beta N$ are constant in different channel bandwidth settings, where the available range of the extraction ratio $\beta$ is determined by the signal size $N$ and the spectrum utilization ratio $\alpha$. Therefore, the proposed scheme can switch among different bandwidth settings.

In the paper, the proposed scheme is tested in the real-world TV signals collected by the RFeye node located at Queen Mary University of London $\left(51.523021^{\circ} \mathrm{N} 0.041592^{\circ} \mathrm{W}\right.$. As observed in Fig. 4 (a), the number of channels $M$ in TVWS is 40 and strong DVB-T signal is received at channel $\{22,23,25,26,28,29,30,33\}$ in the recorded power spectrum. Thus the PU's spectrum utilization ratio is $\alpha=20 \%$.

To obtain each channel occupancy information, we implement the proposed permutation and filtering algorithm to lower the sparsity level of the received signal before spectrum estimation via sFFT.

The time domain permutation $t \longmapsto M t \bmod N$ is firstly applied to the input signal (e.g. Fig. 4 (b)), which corresponds to the spectrum permutation $f \longmapsto M^{-1} f \bmod N$ in the frequency domain. The frequency components from different channels are uniformly interleaved with each other in the frequency domain after the permutation.

The signal size $N$ equals to 819199 in Fig. 4 . We take the nearest $N F F T=2^{20}=1,048,576$ in power of 2 to get its spectrum in sFFT. The empirical sparsity bounds $\kappa$ of sFFT under $2^{20}$ is around 420 . Thus the range of the extraction ratio $\beta$ to retain faster runtime of sFFT than FFT is $\beta \leq \beta_{\max }=$ $0.256 \%$.

Multi-channel filtering is then performed through one bandpass filter with bandwidth $B_{\text {filter }}=\beta B$ to extract the desired number of central frequencies from each channel.

Finally, the frequencies in the filter output are restored to their original places by carrying out a reverse time dilation $t \longmapsto M^{-1} t \bmod N$. The corresponding permutation and filtering in the signal spectrum under the extraction ratio $\beta=0.25 \%$ is illustrated in Fig. 5 .

Signal spectrum is then estimated through sFFT based on its subsampling strategy with faster runtime and low hardware complexity. Based on the estimated spectrum, we jointly decide the presence or absence of PUs on multiple channels. To distinguish a channel occupied by PU signal from a spectrum hole that contains noise only signal, the received signal power on each channel is computed and compared with the threshold dependent on the noise variance of the environment.

If the noise spectrum is assumed to be white, the average noise power can be estimated as

$$
S_{n}=\frac{1}{|\mathcal{W}|} \sum_{k \in \mathcal{W}}\left(\widehat{x}_{k}\right)^{2},
$$

where $\mathcal{W}$ represent the classified noise sets containing the indices of the unoccupied spectral components. As the grey

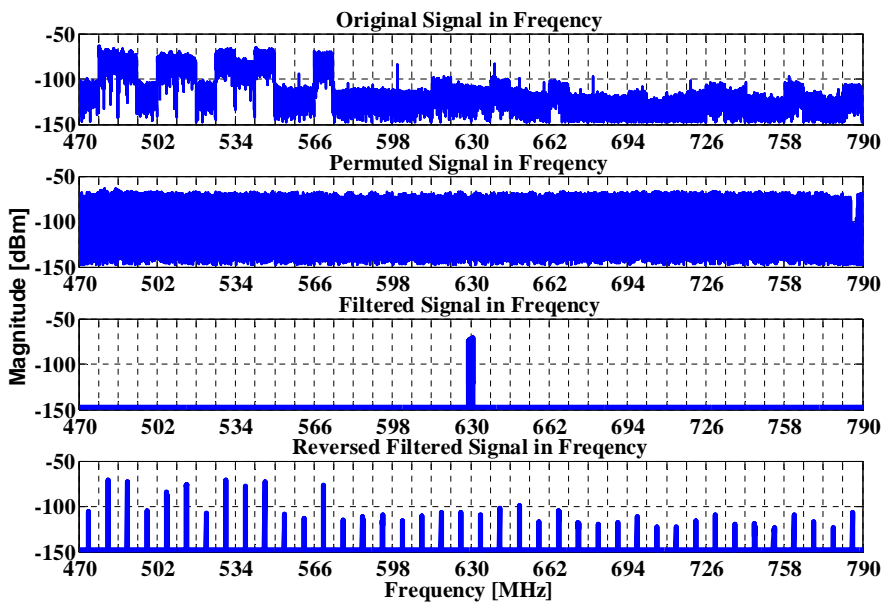

Fig. 5. Permutation and filtering on real-world TVWS signal under $\beta=$ $0.25 \%$.

dash line in Fig. 4 (a) shows, the average noise power $S_{n}$ in the recorded real-time signal is $-121 \mathrm{dBm}$.

However, in practice, real-time noise magnitude fluctuates with time and radio environment as the noise power is susceptible to the interference from other channels or systems and influenced by environmental factors, such as temperature. These noise uncertainties impose fundamental limitations on detection performance. To address this issue, the detection threshold $\lambda$ is set as

$$
\lambda=S_{n}+\eta,
$$

where $\eta$ is the noise uncertainty value that reflects the upper bound for the noise power that can be reached above its estimated value $S_{n}$. Based on the historical data on the noise energy variation range in Fig. 4 (a), the noise uncertainty $\eta$ varies from 0 to $27 \mathrm{~dB}$.

Before real-time spectrum sensing, secondary devices estimate the instantaneous noise variance through a learning process and averages the history statistics to set up the threshold. If the calculated average power of the channel of interest exceeds the corresponding threshold, we declare that a PU signal is present in that channel.

\section{EXPERIMENTAL RESULTS}

In this section, we evaluate the proposed wideband spectrum sensing scheme using both simulated TVWS signals as well as real-time TVWS signals. We discuss the performance evaluation measures we use in the comparison and we analyse and critically discuss the obtained results.

\section{A. Experimental Setup}

Consider the incumbent PU signal that is OFDM simulated, which is used in the DVB-T signal over TVWS. The observed wideband signal ranges from $470 \mathrm{MHz}$ to $790 \mathrm{MHz}$ and has $M=40$ channels in total. Each channel is $8 \mathrm{MHz}$. The spectrum utilization of the input signal is $\alpha$ and $S=\alpha M$ are the channels occupied by PUs. The simulations intend to determine each channel occupancy state, (i.e., occupied or vacant) and to find the positions of the occupied channels. 


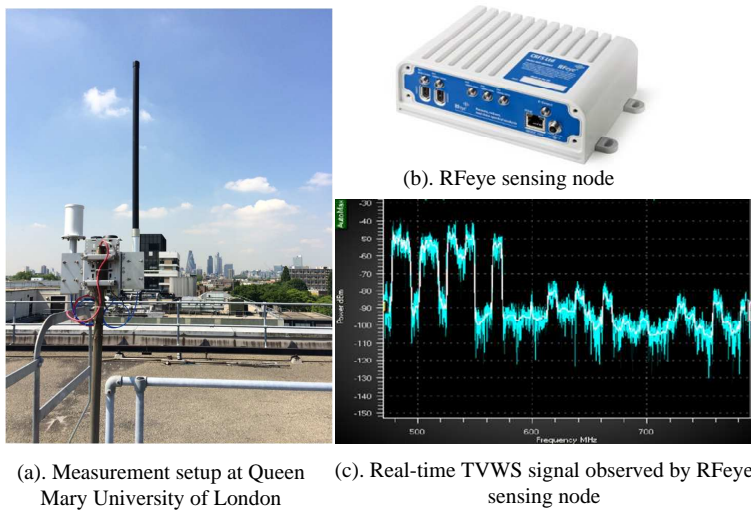

Fig. 6. Measurements setup for real-time TVWS signals recording at Queen Mary University of London.

The real-time TVWS signals are recorded by an RFeye node, an intelligent spectrum monitoring system that can provide real-time $24 / 7$ monitoring of the radio spectrum [46]. To reduce the shadowing of the signal, the RFeye node is located on the top of the Engineering building at Queen Mary University of London $\left(51.523021^{\circ} \mathrm{N} 0.041592^{\circ} \mathrm{W}\right)$. The view from the roof of this building, whose height is about 15 meters above ground, is unobstructed as the average clutter height of surrounding buildings is around 10 meters, as shown in Fig. 6 . There are 40 channels (indexed as Channel 21 - Channel 60) in the recorded TVWS signal, ranging from 470 to $790 \mathrm{MHz}$ and each channel contains either noise only or PU signal with noise, as shown in Fig. 4 (a).

To quantify the detection performance we compute the detection probability $P_{d}$, the fraction of occupied channels correctly being reported as occupied; and the false alarm probability $P_{f}$, the fraction of vacant channels incorrectly being reported as occupied. The target performance requirements for the sensing algorithms are $P_{d} \geq 90 \%$ and $P_{f} \leq 10 \%$ [47].

For spectrum estimation speed evaluation, we compute the runtime of the sFFT and compare it with FFT to see the runtime reduction on different extraction ratios. We also use the average $\mathcal{L}_{1}$ error to compare the difference between the estimated spectrum magnitudes and the original ones for evaluation of spectrum estimation accuracy.

\section{B. Simulated TVWS signal: analysis}

Fig. 7 shows the detection probability $P_{d}$ under different extraction ratios $\beta$ with received SNR distributing from $-20 \mathrm{~dB}$ to $0 \mathrm{~dB}$. The spectrum utilization ratio $\alpha$ is assumed to be $50 \%$, so 20 out of 40 channels are randomly chosen to be occupied. We process the input signal through the proposed permutation and filtering scheme and then implement the conventional multiband joint detection scheme (PFMJD) based on FFT to detect each channel occupancy state. The curves in Fig. 7 are well matched to the theoretical results derived from (9), which supports the theoretical analysis of the proposed permutation and filtering design for multi-channel wideband spectrum sensing. The extraction ratio $\beta=100 \%$ corresponds to the original signal without permutation and filtering. Since the number of samples taken on each channel $L_{m}$ decreases with

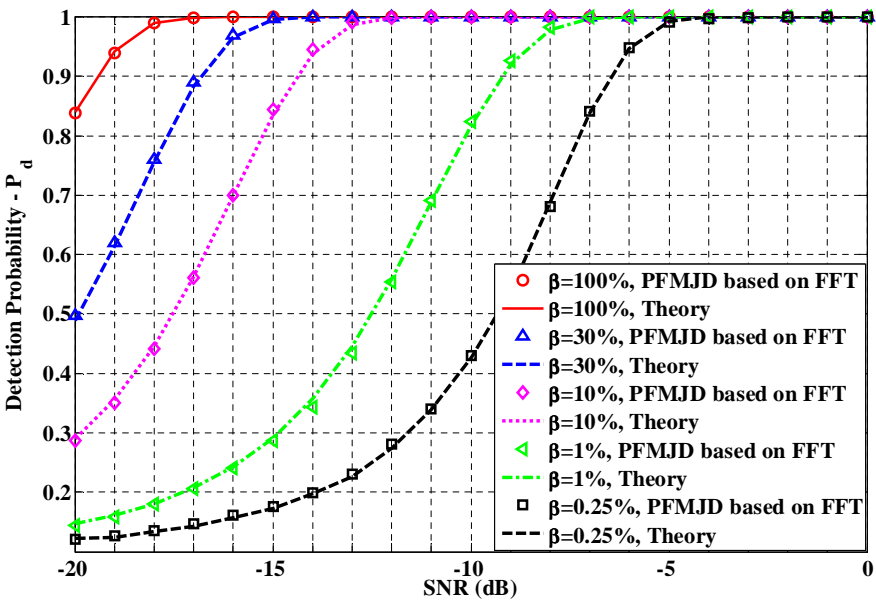

Fig. 7. Detection probability $P_{d}$ vs SNR under different extraction ratio $\beta$, spectrum utilization $\alpha=50 \%$.

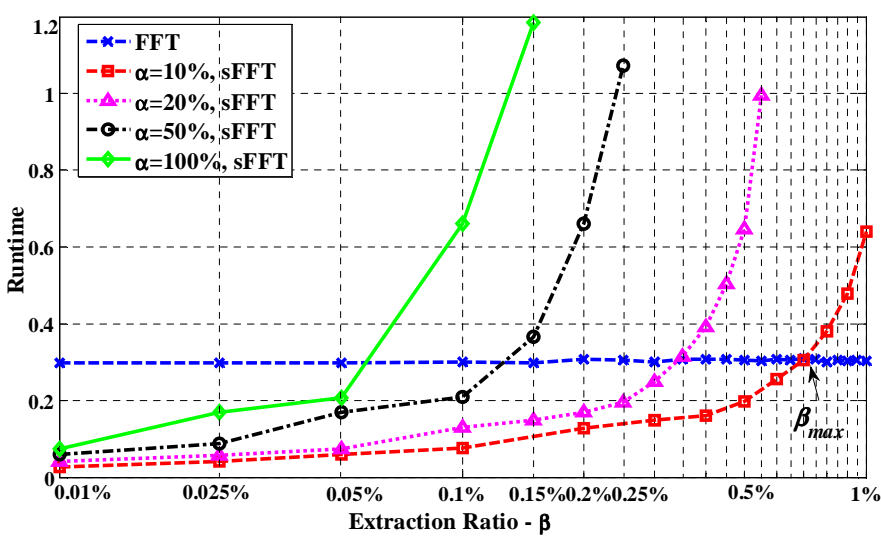

Fig. 8. Runtime of sFFT and FFT for the permuted and filtered signal under different spectrum utilization $\alpha$ and extraction ratios $\beta$.

$\beta$, the detection performance is degraded in the low SNR region, e.g., less than $-5 \mathrm{~dB}$.

Next, we implement sFFT to estimate the spectrum of the permuted and filtered signal for its sub-Nyquist sampling rate and reduced runtime. We begin by evaluating the spectrum computation speed of the proposed wideband sensing scheme based on SFFT. We take the conventional approach based on FFT for comparison [48]. After feeding the input signal into the proposed permutation and filtering algorithm, we compare the runtime of FFT and sFFT. We fix the signal size $N=2^{22}$ and vary the spectrum utilization $\alpha$ and extraction ratio $\beta$, thus current signal sparsity $\kappa^{\prime}=\alpha \beta N$ changes correspondingly.

Through the low extraction ratio $\beta$, sFFT can compute signal spectrum with varying spectrum utilization $\alpha$. Even if the spectrum is highly occupied by PUs, i.e., high $\alpha$ up to $100 \%$, the proposed algorithm can get its spectrum occupancy state using the sub-Nyquist sampling of sFFT based on the proposed permutation and filtering method. As Fig. 8 shows, the runtime of the proposed scheme based on sFFT is sublinear with the spectrum utilization $\alpha$ and extraction ratio $\beta$, while the runtime of FFT is almost constant as $\alpha$ or $\beta$ varies. This is because the runtime of FFT is $O(N \cdot \log N)$, independent of the signal sparsity. Thus, it is more efficient to implement wideband 


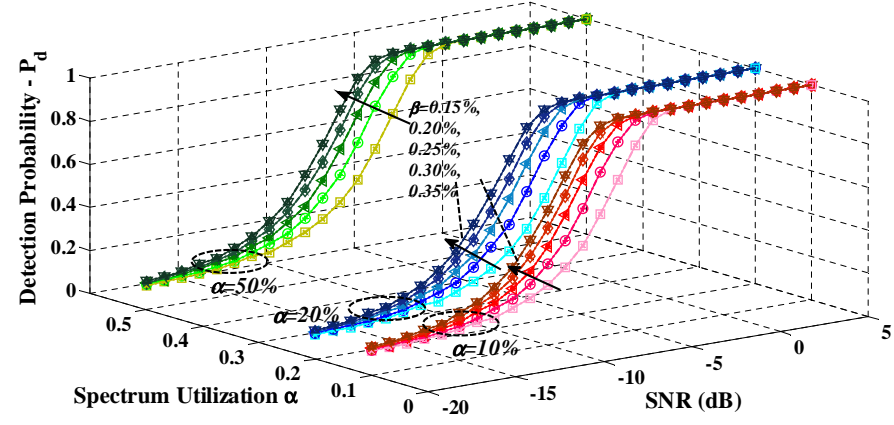

Fig. 9. Detection probability $P_{d}$ vs SNR for the proposed Wideband Sensing scheme and the conventional MJD based on FFT under different extraction ratios $\beta$ with different spectrum utilization $\alpha=10 \%, 20 \%, 50 \%$.

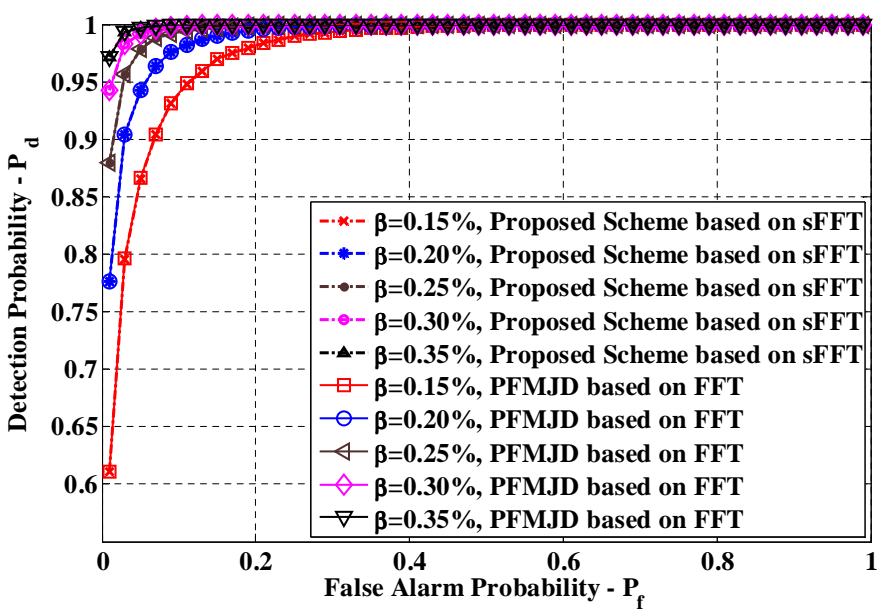

Fig. 10. ROC curve for the proposed Wideband Sensing scheme and the conventional MJD based on FFT under different extraction ratios $\beta$.

sensing through sFFT based on the proposed permutation and filtering algorithm to achieve faster runtime. However, for a given $\alpha$, as extraction ratio $\beta$ increases, the number of samples taken from each channel increases. Therefore, the runtime of sFFT exceeds FFT finally as signal sparsity $\kappa^{\prime}$ increases over sFFT sparsity bound.

Then, we evaluate the detection probability $P_{d}$ the proposed wideband sensing scheme based on sFFT achieved under different SNR and $P_{f}$. We first fix the false alarm probability $P_{f}$ to 0.1 and vary the received SNR at the SU from $-20 \mathrm{~dB}$ to $5 \mathrm{~dB}$. The spectrum utilization ratio $\alpha$ is set to $10 \%, 20 \%$ and $50 \%$. The extraction ratio $\beta$ is set to $0.15 \%, 0.20 \%, 0.25 \%, 0.3 \%, 0.35 \%$. As Fig. 9 presents, the detection probability $P_{d}$ increases as received SNR and extraction ratio $\beta$ increases. For the same extraction ratio $\beta$, the proposed wideband sensing scheme based on sFFT under the proposed permutation and filtering scheme can detect the TVWS signal at comparable performance to the conventional PFMJD scheme. Moreover, the detection performance for different spectrum utilization $\alpha$ is almost the same under same extraction ratio $\beta$. This is because the detection probability $P_{d}$ is only related to the number of samples extracted on each channel $L_{m}=\lfloor\beta L\rfloor$ as (9) shows. Comparing the results from Fig. 8 and Fig. 9, the detection performance of the proposed scheme is independent of spectrum utilization $\alpha$ of the input
TABLE II

IMPROVEMENTS OF SFFT COMPARED WITH FFT WITH SUBSAMPLING RATE $4.00 \%$ UNDER DIFFERENT EXTRACTION RATIO $\beta$

\begin{tabular}{|c|c|c|}
\hline Extraction Ratio $\beta$ & Runtime Reduction & $\mathcal{L}_{1}$ Error \\
\hline $0.15 \%$ & $34.85 \%$ & 0.033 \\
\hline $0.20 \%$ & $16.67 \%$ & 0.032 \\
\hline $0.25 \%$ & $6.067 \%$ & 0.034 \\
\hline $0.30 \%$ & $-4.55 \%$ & 0.032 \\
\hline $0.35 \%$ & $-22.73 \%$ & 0.032 \\
\hline
\end{tabular}

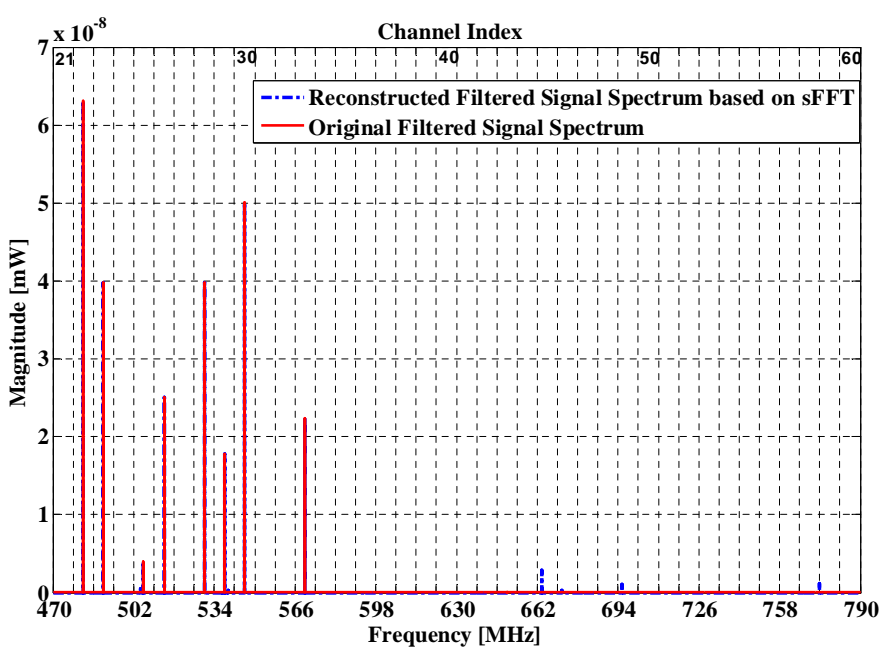

Fig. 11. Comparison of the reconstructed and original filtered signal with extraction ratio $\beta=0.25 \%$.

signal but the available range of $\beta_{\max }$ to retain faster runtime of sFFT shrinks as $\alpha$ increases.

In Fig. 10, we fix the spectrum utilization $\alpha=20 \%$ and simulate the Receiver Operating Characteristic (ROC) curve of the proposed wideband sensing scheme and the PFMJD scheme. The SNR of the SU is set to be $-5 \mathrm{~dB}$ and the false alarm probability $P_{f}$ is varied from 0.01 to 1 . As Fig. 10 shows, as the false alarm probability $P_{f}$ increases, the detection probability $P_{d}$ increases as well. The experiment result shows that the proposed wideband sensing algorithm performs almost the same as the conventional MJD scheme based on FFT under the same extraction ratio $\beta$.

\section{Real-world TVWS signals: analysis}

After the robust performance of the proposed wideband spectrum sensing scheme has been validated with simulated DVB-T signals, we test it on real-world TVWS signals recorded by the RFeye node installed in our lab as shown in Fig. 6. We compare the spectrum estimation preformation of the proposed scheme based on SFFT with the original recorded real-world TVWS signals and analyse the impact of threshold setting in (13) under noise uncertainty.

We implement sFFT to estimate the spectrum of the input signal after the proposed permutation and filtering scheme. The difference between the estimated and original spectrum magnitude is shown with the reconstruction error of the sFFT (the average $\mathcal{L}_{1}$ error of the amplitude estimation). Table II shows the runtime reduction from FFT and $\mathcal{L}_{1}$ error per large magnitude frequency estimation under different extraction ratio $\beta$ when the subsampling rate is reduced to $4 \%$ of 


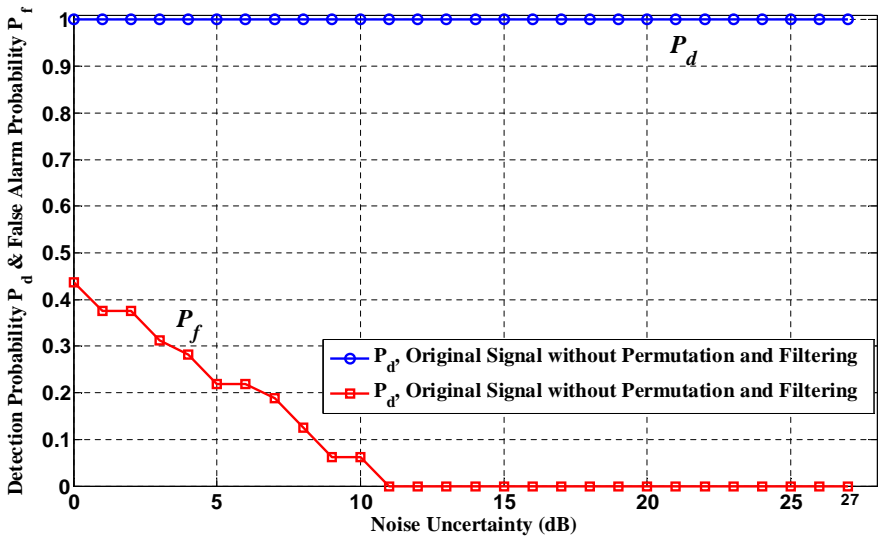

(a)

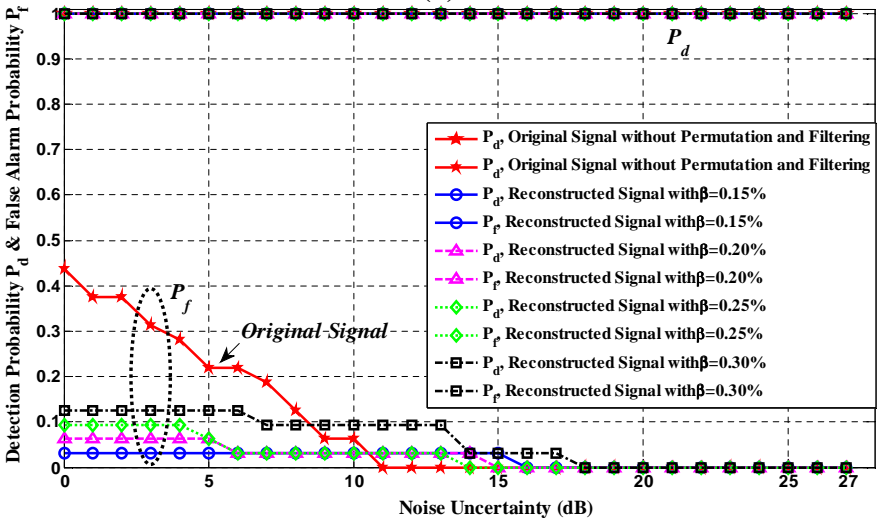

(b)

Fig. 12. Detection probability $P_{d}$ and false alarm probability $P_{f}$ of (a) the original real-world signal detection under noise uncertainty $\eta$ and (b) the reconstructed real-world signal detection with different extraction ratio $\beta$ under noise uncertainty $\eta$.

the Nyquist sampling. When extraction ratio $\beta$ increases to $0.30 \%$, the runtime of sFFT exceeds FFT as signal sparsity $\kappa^{\prime}$ increases over sFFT sparsity bound.

The comparison of the estimated and the original signal spectrum with $\beta=0.25 \%$ (Fig. 11) shows that the proposed scheme based on sFFT can accurately locate the positions of the high magnitude frequencies.

To evaluate the impact of threshold setting in (13) for the recorded real-world TVWS signals under noise uncertainty, we calculate the signal power in each channel and compare it with the threshold. The noise uncertainty is varied from 0 to $27 \mathrm{~dB}$, based on the historical data on the noise energy variation range. The corresponding $P_{d}$ and $P_{f}$ are computed by comparing the experimental results with the measurement results (Fig. 12).

Fig. 12 (a) shows the detection probability $P_{d}$ and false alarm probability $P_{f}$ of the original real-world signal detection under noise uncertainty $\eta$. The signal power in each channel is computed based on its original spectrum without permutation and filtering and sFFT implementation. The false alarm probability $P_{f}$ decreases with the increase of the noise uncertainty $\eta$ (Fig. 12 (a)), because the narrower the margin assumed for the noise to vary over, the higher the probability that a fluctuation in the noise only signal will be falsely interpreted as a PU signal. As noise uncertainty increases, the floating range of noise increases as well. The detection performance $P_{d}$ is expected to degrade with the increase of the noise uncertainty $\eta$. Since the magnitude of real-world PU signal is higher than the upper bound of the instantaneous noise power that can be reached over, $P_{d}$ is constant at $100 \%$ in Fig. 12 (a). The predefined threshold should be larger than $-112 \mathrm{dBm}$, i.e., $\eta>9 \mathrm{~dB}$ for the recorded TVWS signal to retain the target false alarm probability $P_{f}$ to be less than $10 \%$.

We then evaluate the detection performance of the proposed spectrum sensing scheme for the real-world signal under different extraction ratios $\beta$. Fig. 12 (b) shows the detection probability $P_{d}$ and false alarm probability $P_{f}$ for the estimated signal spectrum based on sFFT with different extraction ratios $\beta$. As the number of samples retained on each channel is reduced to $L_{m}=\lfloor\beta L\rfloor$, the probability that the noise fluctuation exceeds the predefined threshed will decrease with such reduced number of samples. Therefore, when the noise uncertainty is less than $8 \mathrm{~dB}$, the reconstructed signal under different extraction ratios achieves smaller $P_{f}$ than that of the original signal. Since signal magnitudes vary randomly in each channel, the power of the extracted subset of the signal is varied randomly as well, thus influencing the corresponding detection performance. When the noise increases to $11 \mathrm{~dB}, P_{f}$ of original signal is decreased to $0 \%$, but $P_{f}$ of reconstructed spectrum is larger than original signal and will decrease to $0 \%$ until $\eta=18 \mathrm{~dB}$. As the desired requirements of $P_{f}$ should be less than $10 \%$, the threshold setting in the original signal $(\eta>9 \mathrm{~dB})$ is suitable to the reconstructed spectrum under different extraction ratio $\beta$.

\section{CONCLUSION}

We presented an approach for wideband spectrum sensing over TVWS. Based on the prior information on the number of channels and input spectrum utilization, the received signal is pre-processed through a permutation and filtering scheme that reduces signal sparsity to implement sFFT on wideband spectrum sensing. As over TVWS the power spectral density is almost flat within each channel, the extracted subset of frequency samples near the central frequency retains the corresponding channel occupancy state in each channel. The proposed scheme achieves (i) sub-Nyquist wideband sensing through permutation and filtering even when the signal is highly occupied by PUs; (ii) faster runtime and low hardware complexity; (iii) high spectrum estimation accuracy with the adopted subsampling strategy to achieve considerable detection performance as the conventional MJD scheme based on FFT at the same extraction ratio. Finally, the proposed algorithm is further evaluated on real-world TVWS signals.

The real-world spectral data will be useful in cognitive radio simulations and ultimately in the development of cognitive radio systems and the regulatory regime that enables these systems [6]. As the proposed wideband spectrum sensing requires prior information on spectrum utilization, dynamic signal sparsity estimation is needed before spectrum sensing. The proposed scheme works for the spectrum with fixed channel bandwidth and flat power spectrum density, such 
as TVWS and LTE. Our future work will address varied bandwidth and power spectrum density, such as the detection of unregistered PMSE users.

\section{REFERENCES}

[1] Y. Ma, Y. Gao, and C. G. Parini, "Sub-Nyquist rate wideband spectrum sensing over TV white space for M2M communications," in Proc. IEEE Int. conf. on World of Wireless, Mobile and Multimedia Netw. (WoWMoM), Boston, MA, Jun. 2015, pp. 1-6.

[2] J. Mitola and G. Maguire JR., "Cognitive Radio: Making software radios more personal," IEEE Pers. Commun., vol. 6, no. 4, pp. 13-18, Aug. 1999.

[3] Office of Commun. (Jul. 2009). Digital dividend: cognitive access. [Online]. Available: http://stakeholders.ofcom.org.uk/binaries/ consultations/cognitive/statement/statement.pdf

[4] H. Sun, A. Nallanathan, C.-X. Wang, and Y. Chen, "Wideband spectrum sensing for cognitive radio network: a survey," IEEE Trans. Wireless Commun., vol. 20, no. 2, pp. 74-81, Apr. 2013.

[5] Office of Communications. (Feb. 2015). Implementing TV White Spaces. [Online]. Available: http://stakeholders.ofcom.org.uk/binaries/ consultations/white-space-coexistence/statement/tvws-statement.pdf

[6] O. Holland, S. Ping, Y. Gao, Z. Qin, A. Aijaz, J. Chareau, P. Chawdhry, and H. Kokkinen, "To white space or not to white space: That is the trial within the ofcom TV white spaces pilot," in Proc. IEEE Int. Conf. on Dynamic Spectrum Access Netw. (DYSPAN), Stockholm, Sweden, Sep. 2015, pp. 11-22.

[7] Y. Gao, Z. Qin, Z. Feng, Q. Zhang, O. Holland, and M. Dohler, "Scalable and Reliable IoT Enabled By Dynamic Spectrum Management for M2M in LTE-A," IEEE Internet of Things Journal, vol. 3, no. 6, pp. 11351145, Dec. 2016

[8] L. Bedogni, A. Trotta, and M. D. Felice, "On 3-dimensional spectrum sharing for TV white and Gray Space networks," in Proc. IEEE Int. conf. on World of Wireless, Mobile and Multimedia Netw. (WoWMoM), Jun. 2015, pp. 1-8.

[9] Y. Ma, Y. Gao, Y.-C. Liang, and S. Cui, "Reliable and Efficient SubNyquist Wideband Spectrum Sensing in Cooperative Cognitive Radio Networks," IEEE J. Sel. Areas Commun., vol. 34, no. 10, pp. 27502762, Oct. 2016.

[10] Z. Qin, Y. Gao, M. Plumbley, and C. Parini, "Wideband spectrum sensing on real-time signals at sub-nyquist sampling rates in single and cooperative multiple nodes," IEEE Trans. Signal Process., vol. 64, no. 12, pp. 3106-3117, Jun. 2016.

[11] Z. Qin, Y. Gao, and C. G. Parini, "Data-assisted low complexity compressive spectrum sensing on real-time signals under sub-nyquist rate," IEEE Trans. Wireless Commun., vol. 15, no. 2, pp. 1174-1185, Feb. 2016.

[12] Z. Quan, S. Cui, A. H. Sayed, and H. V. Poor, "Optimal Multiband Joint Detection for Spectrum Sensing in Cognitive Radio Networks," IEEE Trans. Signal Process., vol. 57, no. 3, pp. 1128-1140, Mar. 2009

[13] Z. Tian and G. B. Giannakis, "A Wavelet Approach to Wideband Spectrum Sensing for Cognitive Radios," in Proc. Int. Conf. on Cognitive Radio Oriented Wireless Netw. and Commun. (CROWNCOM), Mykonos Island, Greece, Jun. 2006, pp. 1-5.

[14] Y. Pei, Y.-C. Liang, K. C. Teh, and K. H. Li, "Energy-Efficient Design of Sequential Channel Sensing in Cognitive Radio Networks: Optimal Sensing Strategy, Power Allocation, and Sensing Order," IEEE J. Sel. Areas Commun., vol. 29, no. 8, pp. 1648-1659, Sep. 2011.

[15] R. Yu, Y. Zhang, L. Yi, S. Xie, L. Song, and M. Guizani, "Secondary users cooperation in cognitive radio networks: balancing sensing accuracy and efficiency," IEEE Wireless Commun., vol. 19, no. 2, pp. 30-37, Apr. 2012.

[16] B. Farhang-Boroujeny, "Filter Bank Spectrum Sensing for Cognitive Radios," IEEE Trans. Signal Process., vol. 56, no. 5, pp. 1801-1811, May 2008.

[17] H. Sun, A. Nallanathan, S. Cui, and C. X. Wang, "Cooperative Wideband Spectrum Sensing Over Fading Channels," IEEE Trans. Veh. Technol., vol. 65 , no. 3 , pp. $1382-1394$, Mar. 2016.

[18] Z. Tian and G. B. Giannakis, "Compressed sensing for wideband cognitive radios," in Proc. IEEE Int. conf. on Acoust., Speech and Signal Process. (ICASSP), vol. 4, Honolulu, HI, Apr. 2007, pp. 1357-1360.

[19] J. Jiang, H. Sun, D. Baglee, and H. V. Poor, "Achieving Autonomous Compressive Spectrum Sensing for Cognitive Radios," IEEE Trans. Veh. Technol., vol. 65, no. 3, pp. 1281-1291, Mar. 2016.
[20] W. Chen and I. J. Wassell, "Optimized Node Selection for Compressive Sleeping Wireless Sensor Networks," IEEE Trans. Veh. Technol., vol. 65, no. 2, pp. 827-836, Feb. 2016.

[21] X. Zhang, Y. Ma, and Y. Gao, "Adaptively Regularized Compressive Spectrum Sensing From Real-time Signals to Real-time Processing,", in Proc. IEEE Global Commun. Conf. (GLOBECOM), Washington, D.C., Dec. 2016 (to appear).

[22] B. Sun, Q. Chen, X. Xu, Y. He, and J. Jiang, "Permuted and filtered spectrum compressive sensing," IEEE Signal Process. Lett., vol. 20 , no. 7, pp. 685-688, Jul. 2013.

[23] Z. Zhang, Z. Han, H. Li, D. Yang, and C. Pei, "Belief Propagation Based Cooperative Compressed Spectrum Sensing in Wideband Cognitive Radio Networks," IEEE Trans. Wireless Commun., vol. 10, no. 9, pp. 3020-3031, Sep. 2011.

[24] Z. Zeinalkhani and A. H. Banihashemi, "Iterative recovery algorithms for compressed sensing of wideband block sparse spectrums," in Proc. IEEE Int. Conference on Commun. (ICC), Ottawa, Canada, Jun. 2012, pp. $1630-1634$.

[25] F. Zeng, C. Li, and Z. Tian, "Distributed Compressive Spectrum Sensing in Cooperative Multihop Cognitive Networks," IEEE J. Sel. Signal Process., vol. 5, no. 1, pp. 37-48, Feb 2011.

[26] E. Candes and T. Tao, "Near-Optimal Signal Recovery From Random Projections: Universal Encoding Strategies?" IEEE Trans. Inf. Theory, vol. 52, no. 12, pp. 5406-5425, Dec. 2006.

[27] J. A. Tropp, J. N. Laska, M. F. Duarte, J. K. Romberg, and R. G. Baraniuk, "Beyond Nyquist: Efficient Sampling of Sparse Bandlimited Signals," IEEE Trans. Inf. Theory, vol. 56, no. 1, pp. 520-544, Jan. 2010.

[28] M. Mishali and Y. C. Eldar, "From Theory to Practice: Sub-Nyquist Sampling of Sparse Wideband Analog Signals," IEEE J. Sel. Signal Process., vol. 4, no. 2, pp. 375-391, Apr. 2010.

[29] H. Hassanieh, L. Shi, O. Abari, E. Hamed, and D. Katabi, "GHz-wide sensing and decoding using the sparse Fourier transform," in Proc. IEEE Int. Conf. on Computer Commun. (INFOCOM), Toronto, CA, Apr. 2014, pp. 2256-2264.

[30] H. Hassanieh, P. Indyk, D. Katabi, and E. Price, "Simple and practical algorithm for sparse Fourier transform," in Proc. of the ACM-SIAM Symp. on Discrete Algorithms, Jan. 2012.

[31] G. Forney, "Maximum-likelihood sequence estimation of digital sequences in the presence of intersymbol interference," IEEE Trans. Inf. Theory, vol. 18, no. 3, pp. 363-378, May 1972.

[32] Y.-C. Liang, Y. Zeng, E. C. Peh, and A. T. Hoang, "Sensing-Throughput Tradeoff for Cognitive Radio Networks," IEEE Trans. Wireless Commun., vol. 7, no. 4, pp. 1326-1337, Apr. 2008.

[33] A. Tani and R. Fantacci, "A Low-Complexity Cyclostationary-Based Spectrum Sensing for UWB and WiMAX Coexistence With Noise Uncertainty," IEEE Trans. Veh. Technol., vol. 59, no. 6, pp. 2940-2950, Jul. 2010

[34] X. Ying, J. Zhang, L. Yan, G. Zhang, M. Chen, and R. Chandra, "Exploring Indoor White Spaces in Metropolises," in Proc. IEEE Int. Conf. on Computer Commun. (INFOCOM), Hong Kong, Apr. 2015, pp. $47-48$.

[35] J. Ribeiro et al., "Testbed for combination of local sensing with geolocation database in real environments," IEEE Wireless Commun., vol. 19 , no. 4, p. 5966, Aug. 2012.

[36] T. M. Taher, R. B. Bacchus, K. J. Zdunek, and D. A. Roberson, "Longterm Spectral Occupancy Findings in Chicago," in Proc. IEEE Int. Conf. on Dynamic Spectrum Access Netw. (DYSPAN), Aachen, May 2011, pp. 100-107.

[37] M. Lopez-Benitez, A. Umbert, and F. Casadevall, "Evaluation of spectrum occupancy in spain for cognitive radio applications," in VTC Spring 2009 - IEEE 69th Vehicular Technology Conference, Apr. 2009, pp. 1-5.

[38] S. Verdu, "Minimum probability of error for asynchronous Gaussian multiple-access channels," IEEE Trans. Inf. Theory, vol. 32, no. 1, pp. 85-96, Jan 1986.

[39] A. Ebrahimzadeh, M. Najimi, S. Andargoli, and A. Fallahi, "Sensor Selection and Optimal Energy Detection Threshold for Efficient Cooperative Spectrum Sensing," IEEE Trans. Veh. Technol., vol. 64, no. 4, pp. $1565-1577$, Apr. 2015

[40] M. Derakhshani, T. Le-Ngoc, and M. Nasiri-Kenari, "Efficient Cooperative Cyclostationary Spectrum Sensing in Cognitive Radios at Low SNR Regimes," IEEE Trans. Wireless Commun., vol. 10, no. 11, pp. 3754-3764, Nov. 2011.

[41] P. Paysarvi-Hoseini and N. C. Beaulieu, "On the Benefits of Multichannel/Wideband Spectrum Sensing with Non-Uniform Channel Sensing Durations for Cognitive Radio Networks," IEEE Trans. Commun., vol. 60 , no. 9 , pp. 2434-2443, Sep. 2012. 
[42] H. Hassanieh, F. Adib, D. Katabi, and P. Indyk, "Faster GPS via the Sparse Fourier Transform," in Proc. Annu. Int. Cof. on Mobile computing and networking(ACM MOBICOM), Aug 2012, pp. 353-364.

[43] W. Kester, Mixed-signal and DSP Design Techniques, 1st ed. Oxford: Newnes, 2003.

[44] G. B. Arfken, H. J. Weber, and F. E. Harris, Mathematical Methods for Physicists, 7th ed. San Diego: Academic Press, 2001.

[45] R. Tandra and A. Sahai, "SNR Walls for Signal Detection," IEEE Journal of Selected Topics in Signal Processing, vol. 2, no. 1, pp. 4-17, Feb. 2008.

[46] CRFS. (Oct. 2015). RFeye Node. [Online]. Available: https://uk.crfs. com/zh-hans/products/rfeyenode/node-20-6/

[47] "IEEE Standard for Wireless Regional Area Networks Part 22: Cognitive Wireless RAN MAC and PHY specifications: Policies and procedures for operation in the TV Bands," IEEE Std. 802.22-2011, pp. 1-680, Jul. 2011.

[48] M. Frigo and S. G. Johnson. FFTW 3.2.3. [Online]. Available: http://www.fftw.org/ 